\title{
Performance Test of Double-Disk Type Output Window of 28/35 GHz Dual-Frequency Gyrotron for CW Operation*)
}

\author{
Maki OKADA, Tsuyoshi KARIYA, Tsuyoshi IMAI, Ryutaro MINAMI, Tomoharu NUMAKURA, \\ Yuto EBASHI, Fumiya MOTOYOSHI and Yousuke NAKASHIMA \\ Plasma Research Center, University of Tsukuba, Tsukuba, Ibaraki 305-8577, Japan
}

(Received 29 September 2018 / Accepted 25 December 2018)

\begin{abstract}
A $28 \mathrm{GHz} / 35 \mathrm{GHz}$ dual-frequency gyrotron has been developed for use by the University of Tsukuba and its collaborative research institutions. This gyrotron incorporates a sapphire double disk window for $28 \mathrm{GHz}$ 0.4 MW-Continuous Wave (CW) operation. The heat transfer coefficient was estimated by comparing the measurement results of the output window temperature with calculated values and the results suggest that this gyrotron can operate in the $\mathrm{CW}$ operation.
\end{abstract}

(c) 2019 The Japan Society of Plasma Science and Nuclear Fusion Research

Keywords: gyrotron, ECH system, millimeter wave, $28 \mathrm{GHz}, 35 \mathrm{GHz}, \mathrm{CW}$ operation, double-disk window

DOI: $10.1585 / \mathrm{pfr} .14 .2405043$

\section{Introduction}

Gyrotron is mainly used as high-power millimeter wave source for electron cyclotron heating $(\mathrm{ECH})$ and electron cyclotron current drive (ECCD) in many magnetic confinement plasma experimental devices.

High power gyrotrons are currently being developed for fusion research at the Plasma Research Center (PRC) of the University of Tsukuba [1]. In the cases of the GAMMA 10/PDX at the PRC and the National Spherical Torus Experiment Upgrade (NSTX-U) at Princeton Plasma Physics Laboratory (PPPL), a $28 \mathrm{GHz}$ gyrotron which operating with a $1.5-2 \mathrm{MW}$ output that is operational for several seconds is required. The Heliotron $\mathbf{J}$ at the Kyoto University needs a gyrotron that operates at a frequency of $35 \mathrm{GHz}$ with a $1 \mathrm{MW}$ output for one second. Moreover, the Qshu University Experiments with Steady-State Spherical Tokamak (QUEST) ECH system conducted at the Kyushu University requires a $0.4 \mathrm{MW}$-continuous wave $(\mathrm{CW}) \mathrm{gy}$ rotron that operates at $28 \mathrm{GHz}$. In a collaborative investigation, we developed a $28 / 35 \mathrm{GHz}$ dual-frequency gyrotron to achieve the expected performance requirements. The first experimental test was performed on the dualfrequency gyrotron began in 2016 [2] and is ongoing, producing output powers of $1.25 \mathrm{MW}$ at $28 \mathrm{GHz}$ and $0.48 \mathrm{MW}$ at $35 \mathrm{GHz}$. Figures 1 (a) and (b) show the photographic image and the structural cross-section view of the $28 / 35 \mathrm{GHz}$ dual frequency gyrotron. The design parameters of this gyrotron are summarized in Table 1. A triode magnetron injection gun (MIG) is used to control the electron beam for each frequency by optimizing the anode-cathode voltage. The cavity oscillation modes are the $\mathrm{TE}_{8,5}$ mode for $28 \mathrm{GHz}$ and in the $\mathrm{TE}_{10,6}$ mode for $35 \mathrm{GHz}$ in circular

author'se-mail: okada_maki@prc.tsukuba.ac.jp

*) This article is based on the presentation at the 12th International Conference on Open Magnetic Systems for Plasma Confinement (OS2018). (a)
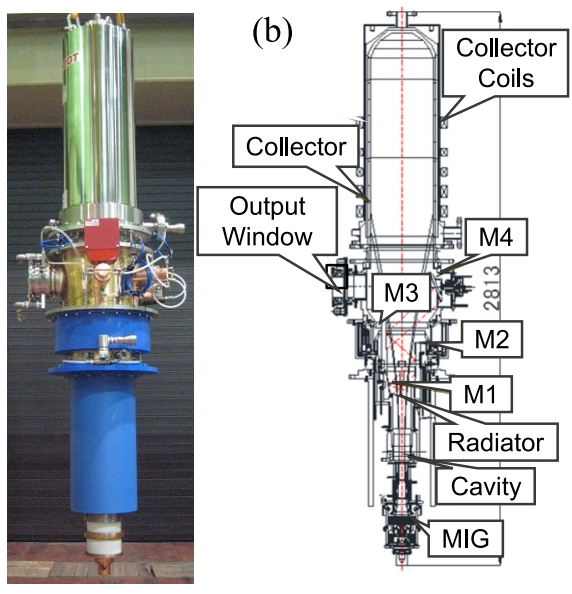

Fig. 1 (a) Photograph and (b) structural cross-section view of $28 / 35 \mathrm{GHz}$ dual-frequency gyrotron.

Table 1 Design specifications of the $28 / 35 \mathrm{GHz}$ dual-frequency gyrotron.

\begin{tabular}{|c|c|c|}
\hline Frequency & $28 \mathrm{GHz}$ & $34.77 \mathrm{GHz}$ \\
\hline Output Power & 1.5-2 MW 0.4 MW & $1 \mathrm{MW}$ \\
\hline Pulse Length & $3 \mathrm{sec} \quad \mathrm{CW}$ & $3 \mathrm{sec}$ \\
\hline Total Efficiency & \multicolumn{2}{|c|}{$50 \%$ (with CPD) } \\
\hline Beam Current & $80 \mathrm{kV} \quad 70 \mathrm{kV}$ & $80 \mathrm{kV}$ \\
\hline Beam Voltage & $20 \mathrm{~A}$ & $40 \mathrm{~A}$ \\
\hline MIG & \multicolumn{2}{|c|}{ Triode } \\
\hline Cavity mode & $\mathrm{TE}_{8,5}$ & $\mathrm{TE}_{10,6}$ \\
\hline Output mode & \multicolumn{2}{|c|}{ Gaussian like } \\
\hline Output Window & \multicolumn{2}{|c|}{ Sapphire double-disk } \\
\hline Radiator & \multicolumn{2}{|c|}{ Build-in } \\
\hline Collector & \multicolumn{2}{|c|}{ CPD (depressed collector) } \\
\hline
\end{tabular}


waveguide. These modes are oscillated by the interaction of electron beam with the RF field in the cavity and converted to a Gaussian-like RF beam using a radiator. The RF beam is transmitted using four mirrors shown as M1 - M4 (Fig. 1 (b)) and is emitted with MW power from the output window which is a sapphire double-disk window (DDW) for $\mathrm{CW}$ operation. The spent electron beam is collected in a collector. The unused parallel kinetic energy of the spent electron beam after participating in the interaction process is recovered by depressing the voltage between the cavity and the collector section (collector potential depression (CPD)).

In this report, we present the performance test results of the output window aiming for $28 \mathrm{GH}-0.4 \mathrm{MW}-\mathrm{CW}$ operations. Its output power will be two times higher than the achieved output power with the previous $28 \mathrm{GHz}-\mathrm{CW}$ gyrotron. The characteristic of the output window as a determining factor to performance of the gyrotron is discussed in Sec. 2. The performance test results are presented in Sec. 3, whereas discussion and conclusion are provided in Sec. 4.

\section{Output Window for CW Operation}

An output window acts as a barrier between the vacuum side of the gyrotron and the transmission line. Highfrequency gyrotron operating in the MW range generally utilize chemical vapor deposition (CVD) diamond [3,4]. A CVD diamond is an excellent window material that has a low loss tangent, high thermal conductivity, and high mechanical strength. However, the diamond disk with the thickness of $8.99 \mathrm{~mm}$ to satisfy the frequency matching condition $(4 \lambda / 2$ for $28 \mathrm{GHz}$ and $5 \lambda / 2$ for $35 \mathrm{GHz}$ : where $\lambda$ is the wavelength in the dielectric) and the large diameter of about $130 \mathrm{~mm}$ due to the large divergence of RF beam is required for the long wavelength gyrotron. Therefore, we used a sapphire as the $28 / 35 \mathrm{GHz}$ dual-frequency gyrotron window material to avoid the high cost and manufacturing complexity associated with CVD diamond.

The power density of the RF beam is highest at the center of the output window since the beam is Gaussianlike. The output window absorbs a portion of the RF power through dielectric loss, while the cooling structure of the window prevents the thermal damage. In the case of the sapphire single-disk window, the coolant cools the peripheral of the sapphire disk. Therefore, the temperature at the center of the sapphire window is not saturated because of its large diameter and low thermal conductivity during $\mathrm{CW}$ operation. Figure 2 shows the temperature rise on the sapphire single-disk window for one-dimensional nonsteady state heat conduction equation solved using a finite different method [5]. The result shows that the temperature of the sapphire single-disk window with a thickness of $3.49 \mathrm{~mm}$ and a radius of $68 \mathrm{~mm}$ is not saturated at $60 \mathrm{~s}$ under $28 \mathrm{GHz}-0.4 \mathrm{MW}$ operation (the temperature of the cooled edge of sapphire disk is $24.5^{\circ} \mathrm{C}$ ). Thus, the gyrotron

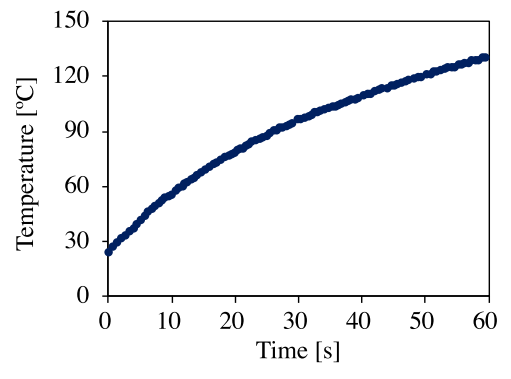

Fig. 2 Calculated temperature of sapphire single-disk window at $28 \mathrm{GHz}-0.4 \mathrm{MW}-\mathrm{CW}$ operations (window thickness: $3.49 \mathrm{~mm}$, radius: $68 \mathrm{~mm}$ ).

(a)
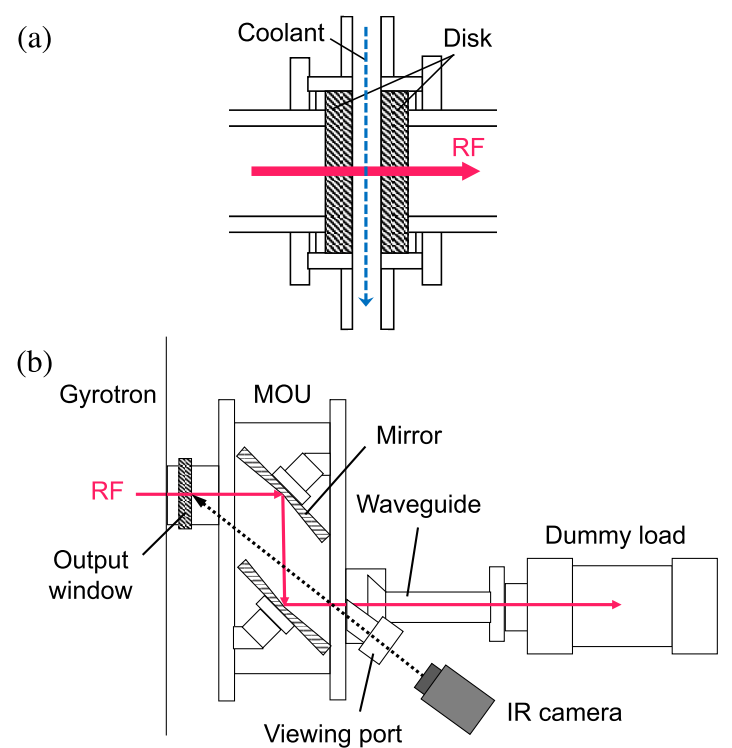

Fig. 3 Schematic diagram of (a) double-disk type out-put window and (b) experimental setup.

adopts the sapphire DDW for CW operation (Fig. 3 (a)).

The coolant of the DDW flows between the two sapphire disks. A fluorocarbon is used as the coolant because of its low RF absorption. A DDW has a large cooling surface and a short thermal transmission path, so the cooling efficiency is significantly improved compared to a singledisk window. The inlet and outlet structures of the coolant are the rectangular to obtain a uniform flow and a large flow rate. In the case of the DDW, the cooled surface temperature of the window should be kept below the boiling point of the fluorocarbon coolant (FC-3283, about $128^{\circ} \mathrm{C}$ ) during $\mathrm{CW}$ operation.

A single crystal C-plane sapphire substrate is used for the output window of the $28 / 35 \mathrm{GHz}$ dual-frequency gyrotron. Since the physical properties such as the dielectric constant and the coefficient of thermal expansion are isotropic on the C-plane. On other planes, these physical properties are direction-dependent. The angle-dependence of the power of the transmitted wave was measured to ex- 
amine the characteristics of the C-plane transmission by the cold testing. The sapphire disk was confirmed to be C-plane from the results of no angle-dependence and the transmission efficiency of about $100 \%$.

Frequency characteristics of a DDW depend on the thickness and permittivity of the sapphire disk and fluorocarbon coolant. The thickness of each sapphire disk is $6.98 \mathrm{~mm}$, which has the frequency matching at both 28 and $35 \mathrm{GHz}$. For the hot test at $28 \mathrm{GHz}$, the frequency matching of the DDW was adjusted by varying the thickness of the coolant layer. The optimum coolant thickness was $4 \mathrm{~mm}$.

\section{Experimental Measurements and Performance Test Results}

A schematic diagram of the experimental setup is shown in Fig. 3 (b). The dummy load coolant absorbs the RF beam power and the output power is calorimetrically measured from the temperature rise of the dummy load coolant. The matching optics unit (MOU) adjusts the profile and phase of the output Gaussian-like RF beam, and this beam is coupled to the $\mathrm{HE}_{11}$ mode in a corrugated waveguide. This $\mathrm{MOU}$ has a viewing port where a $\mathrm{BaF}_{2}$ window is mounted. The center of the output window was observed during the operation of the gyrotron from the left downward direction by IR camera thorough the viewing port because the infrared rays can transmit through the $\mathrm{BaF}_{2}$ window. The time variation of the temperature rise at the center of the output window was measured using an infrared (IR) camera through the port. The temperature of IR camera was calibrated in the same experimental setup shown in Fig. 3 (b) by using a known and steady-state sapphire window temperature, which is equal to the controlled coolant temperature, in the absence of an output power.

Temperature changes at the center of the output window were recorded for operation at $0.45 \mathrm{MW}-2 \mathrm{~s}$ at $28 \mathrm{GHz}$. Figure 4 shows the temperature profiles for each coolant flow rates of $10,20,30,40$, and $50 \mathrm{~L} / \mathrm{min}$. The initial temperature of the window was $20^{\circ} \mathrm{C}$, and the tem-

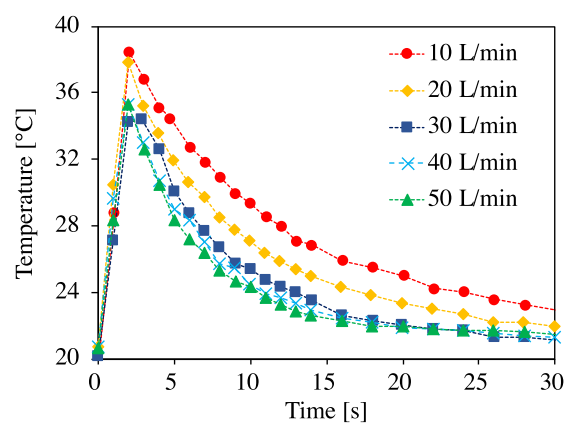

Fig. 4 Time evolutions of the measured output window temperature for the coolant flow rate of $10,20,30,40$, and $50 \mathrm{~L} / \mathrm{min}$ perature increased linearly during the gyrotron operation. After $2 \mathrm{~s}$, the window temperature decreased because the RF power was turned off. An increase in the coolant flow rate resulted in a corresponding reduction in the thermal time constant.

\section{Discussion and Conclusion}

The heat transfer coefficient was obtained by comparing the cooling time obtained experimentally with the numerical results, and the performance of the window was evaluated. When assuming azimuthal symmetry, time evolutions of the temperature of the output window are determined using the 2-dimensional non-steady-state heat conduction equation [5]:

$$
d \rho C_{\mathrm{p}}\left[\frac{\partial T}{\partial t}\right]_{z}=d k \nabla^{2} T+A P(r)-h\left(T-T_{\mathrm{c}}\right),
$$

where $T, d, \rho, C_{\mathrm{p}}, k$, and $P(r)$ are the temperature, thickness, density, specific heat, thermal conductivity, and RF power distribution of the window, respectively. The variables $h$ and $T_{\mathrm{c}}$ represent the surface transfer coefficient and the environmental temperature, respectively. For small loss tangent $\left(<10^{-3}\right)$ and near resonant thickness $(\approx n \lambda / 2)$ of the window disk, the power absorption coefficient is given by [5]:

$$
A=\pi f(\varepsilon+1) \tan \delta d / c,
$$

where $f, \varepsilon, \tan \delta$, and $c$ are the RF frequency in free space, dielectric constant, loss tangent of the window material, and the velocity of light in free space, respectively. The $h\left(T-T_{\mathrm{c}}\right)$ term in Eq. (1) is a cooling term, and the value of $h$ is unknown. The cooling efficiency improves as the value of $h$ increases.

Equation (1) was numerically solved by using a finite difference method, considering the heat generation of the sapphire disk by Gaussian RF beam and heat transfer from the cooled surface of the sapphire disk to the heat sink of the constant temperature $\left(T_{\mathrm{c}}=20^{\circ} \mathrm{C}\right)$. The values of $h$ were estimated by comparing the calculation results with

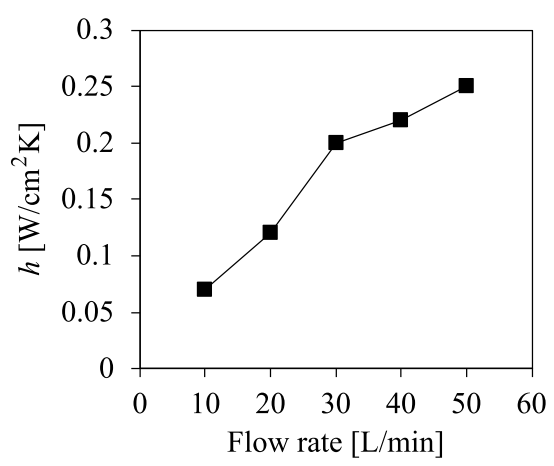

Fig. 5 The value of $h$ obtained for each coolant flow rate. 


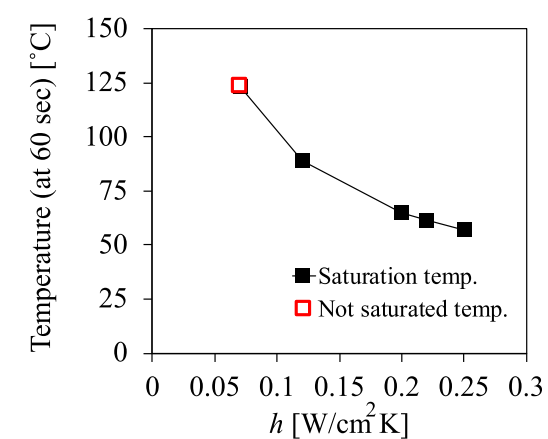

Fig. 6 The output window temperature using each obtained $h$ at $60 \mathrm{~s}$ in the case of operating $28 \mathrm{GHz}-0.4 \mathrm{MW}-\mathrm{CW}$.

the experimental measurements in $2 \mathrm{~s}$ or later. Figure 5 shows the calculated $h$ for each coolant flow rate. This value increases almost linearly with the coolant flow rate.

The time evolutions of the output window temperature during $28 \mathrm{GHz}-0.4 \mathrm{MW}-60 \mathrm{~s}$ operation was calculated for each $h$. Figure 6 shows the $h$ dependency of the window temperature at $60 \mathrm{~s}$. The open symbol indicates that the temperature at $10 \mathrm{~L} / \mathrm{min}\left(h=0.07 \mathrm{~W} / \mathrm{cm}^{2} \mathrm{~K}\right)$ is not saturated at $60 \mathrm{~s}$. The closed symbols indicate that the temperature at $20-50 \mathrm{~L} / \mathrm{min}\left(h=0.12-0.25 \mathrm{~W} / \mathrm{cm}^{2} \mathrm{~K}\right)$ is saturated below the boiling point of the coolant. The cooling efficiency improved as the coolant flow rate increased. It is necessary to increase the pressure of the coolant to acquire the flow rate. The reflection of the RF beam may increase due to the deflection of the sapphire disk by the higher pressure of the coolant. Thus, estimation of the optimum flow rate for $\mathrm{CW}$ operation is needed. The saturation temperature does not change significantly when the $h$, is the greater or equal to $0.2 \mathrm{~W} / \mathrm{cm}^{2} \mathrm{~K}$ or greater. Therefore, $30 \mathrm{~L} / \mathrm{min}\left(h=0.2 \mathrm{~W} / \mathrm{cm}^{2} \mathrm{~K}\right)$ is the optimum value considering safe operation using the present window structure.

The result of the DDW experiment, which optimized the coolant flow rate, suggests that $0.4 \mathrm{MW}-\mathrm{CW}$ operations at $28 \mathrm{GHz}$ will be possible. The next step of this research is a demonstration of quasi-CW operation (e.g., $30 \mathrm{~s}$ ) of the gyrotron, including the verification of the window performance.

\section{Acknowledgment}

The authors thank the members of the GAMMA 10 group of the University of Tsukuba for their collaboration and valuable discussion during this study. This work is partially supported by the NIFS Collaborative program (NIFS16KUGM106, COD29746) and the grant-inaid for scientific research from the Ministry of Education, Science, Sports and Culture of Japan (26249141 and 15H05770B).

[1] T. Imai, et al., Proc. 23rd IAEA Fusion Energy Conf. (Daejeon, 2010), IAEA-CN-165/FTP/P6-12 (2010).

[2] T. Kariya et al., Nucl. Fusion 57, 066001 (2017).

[3] A. Kasugai et al., Rev. Sci. Instrum. 69, No.5, 2160 (1998).

[4] A. Kasugai et al., Nucl. Fusion 48, 054009 (2008).

[5] V.V.P. Singh, et al., J. Infrared Millim. Terahz. Waves 19, No.11, 1451 (1998). 